\title{
The Development of a Paramics Web External Interface - PWeb
}

\author{
Li WEIGANG, Andrew MACIVER, Xiaoxia LI, Jörg DENZINGER, Yaeko YAMASHITA and \\ Hui WANG
}

\begin{abstract}
This paper describes the development of a Web external interface (PWeb) to link the dynamic traffic control simulator - Paramics with real traffic controllers. The Webbased interface is used to administrate the access of multiple controllers to Paramics SNMP external interface during the simulation process, to prevent conflicts among the controllers and to improve the efficiency of the usage of Paramics data input/output. A rule-based system and the connection to the database were developed to arrange the priority of the data to access Paramics. The proposed Web interface has a potential to be applied in on-line simulations to calibrate Paramics microscopic traffic simulation models.
\end{abstract}

Keywords - API, Interface, Paramics, Simulation, Web

\section{INTRODUCTION}

$\mathrm{P}$ ARAMICS (Parallel Microscopic Simulator) is a road traffic microsimulation model using PC or UNIX platforms. It has been widely used for modeling transportation systems throughout the world [1, 2]. Recently, the SIAS version of Paramics has adopted SNMP (Simple Network Management Protocol) as an external interface [3], allowing the software to interact with traffic signal detectors and other traffic control hardware. With this interface, it is possible for the external software and controllers to communicate with a running Paramics simulation, to extract data from it and to adjust parameters within it. An additional useful benefit for this interface would be to use the Internet to connect to traffic control systems on-line.

During the application of the Paramics SNMP external interface, it was found that some aspects within that interface can be further enhanced, such as:

This work was supported in part by $\mathrm{CNPq}$, a science and technology foundation of Brazilian government under the contracts no. 200923/01-3 and 4772113/01-3. It was also supported in part by the University of Calgary, Canada.

L. WEIGANG is with the University of Brasilia, CIC/UnB, C.P. 4466, Brasilia-DF, CEP: 70919-970, Brazil (telephone: 5561 3072702, e-mail: weigang@unb.br). In 2002-2003, he was with the University of Calgary.

A. MACIVER is with the University of Calgary, Canada, (telephone: 1403 2206713, e-mail: amaciver@ucalgary.ca).

J. DENZINGER is with the University of Calgary, Canada, (telephone: 1 403 2205574, e-mail: denzinge@cpsc.ucalgary.ca).

Y. YAMASHITA is with the University of Brasilia (telephone: 5561 3072714, e-mail: yaeko@unb.br).
- transportation engineers using Paramics still have difficulties in using the SNMP external interface if they do not have any SNMP and Visual Basic knowledge;

- the SNMP external interface allows any number of controllers to access Paramics but there is currently no appropriate way to manage multiple controllers, which communicate with Paramics, in order to prevent conflicts [3];

- some data abstraction can be time consuming, for example, log traces of vehicle paths and properties with specific origins and destinations;

- Urban Traffic Control (UTC) systems can utilize other sources of information, which currently cannot be connected via the SNMP external interface.

For example, to manage the controllers, the software developers' warn that: "multiple controller type instances require careful planning and control to prevent conflicts. It is therefore the users' responsibility to ensure the SNMP controllers operate Paramics in an appropriate manner [3].” In the future many cities may use microsimulation for modelling urban traffic control systems, which may involve interacting with numerous traffic controllers. In order to manage the accessibility of Paramics to these controllers the interface between them will become a key issue of on-line simulation and control. The main motivation of this research was to develop a Web external interface to manage the exchange of data between the controllers and Paramics.

There already have been some works dealing with using the Internet in transportation applications. An Internet based system [10] was developed to integrate data, models and users for transportation planning, engineering and operations. In [11], a Control Centre and user interfaces were developed for an urban bus transportation system. The predicted bus's arrival time at any bus stop can be displayed on the Web for the users. More recently, an Intelligent Transportation Web Service was designed with potential applications in traffic information services, logistic service providers, tour and travel services, medical and emergency managements, etc [12].

In this paper, the Web external interface is proposed to connect real traffic controllers with the Paramics SNMP external interface and other users using the Internet (or Web for short). With the Web-based interface, it is possible to prevent conflicts among the controllers and to improve the efficiency of the processing of the input/output data by Paramics. A rule-based system and the connection to the database were developed in this interface to arrange the priority of the data to access Paramics. It should be mentioned that $\mathrm{PWeb}$ was developed not as a substitute for the 
SNMP external interface, but to complement its function and to make the Paramics SNMP external interface more efficient. The Web/Database system in PWeb is used as a bridge to connect the UTC and Paramics. The paper describes an implementation and computer simulation of PWeb, which was developed using an on-line simulation in Calgary.

\section{Prototype of PWeB}

\section{A. Basic structures of the actual and proposed systems}

The SNMP external interface was developed by SIAS [3] and enables Paramics to connect with external hardware, such as signal controllers. The interface allows the traffic modeler to interact with traffic control in a running simulation. Information from the vehicle detectors within the Paramics model can be transmitted via the SNMP external interface to enable a modeler to control signal phase orders and timings, with the option of doing so in real time. Access to the SNMP interface facilitates the rapid development of signal plans (replacing the current signal plans language) and allows flexible use of loop detector data. For example, the interface could be used to link Paramics to UTC systems (including traffic detectors as in this research) or to test innovative control strategies. Through SNMP interfaces, Paramics can test and evaluate the effect of proposed network management control systems, and is a powerful tool when used under constantly changing route choice conditions. Figure 1 shows the basic structure of the Paramics SNMP external interface.

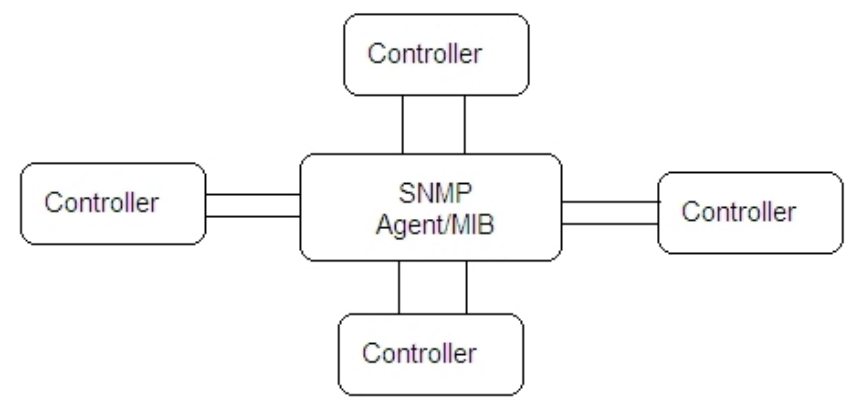

Fig.1. Paramics SNMP external interface structure

The Paramics SNMP external interface allows any number of controllers to access Paramics but there is no appropriate way to manage multiple controllers, which communicate with Paramics and prevent conflicts between them at this moment [3]. In a large urban area, the proposed transportation simulation system may need to connect with a considerable number of controllers. In this research, the study area in downtown Calgary has a total of 320 detectors installed at intersections and road links within this area. This is equivalent to more than 80 controllers. It is necessary to develop a system between the Paramics SNMP external interface simulator and the controllers to manage the information accessing. It is proposed that PWeb will serve this purpose.

By making use of the Internet, which allows access from nearly everywhere, any controller can transmit data to the PWeb database without losing data. The conflict problem can be resolved using properties of existing Web technology. PWeb can manage the transfer and access of the data to the SNMP external interface and then to Paramics during the simulation according to a given priority mechanisms, which is defined by rules. The general idea is to develop a management system with Web/Database as a special controller. The system has been named PWeb (Paramics Web) external interface. The basic structure of it is shown in Figure 2.

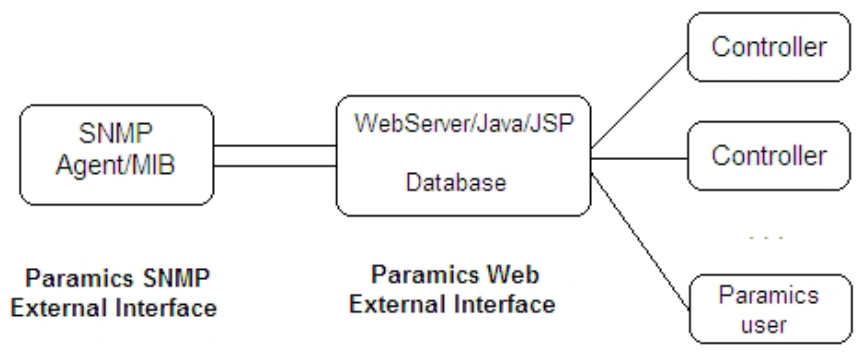

Fig. 2. PWeb structure

\section{B. Communication with Paramics using PWeb}

Three possible ways are proposed for PWeb to communicate with Paramics:

- As a special controller: PWeb can be configured as a controller, which can connect with the Agent/MIB of the Paramics' SNMP external interface. With this configuration, the developed system can properly manage the multiple controllers communicating with Paramics and prevent conflicts. The incoming data from controllers is stored in the PWeb database in a form similar to the original controller. Some rules have to be designed establishing the priorities of the controllers (depending on the location and characters of the events, etc). The data can then be forwarded to Paramics based on these rules. In this way, PWeb can manipulate the data from multiple data sources and then transfer it in a suitable manner to Paramics. Note that by using a set of rules (and a rule interpreter) it is very easy to customize Pweb to different simulations.

- As a common user: PWeb can read/write data from/to the server computer running Paramics. In the input process, the user can input data modifications from everywhere in the Web, then PWeb transfers the data to text files such as snap.* files. On the other hand, as output process, PWeb can also get the simulation results, such as the files from the ../Log/run-00n directory of the Paramics host computer and displays them on a Web page, again making them accessible from everywhere. In a traffic simulation center, the Paramics model can be very busy during a simulation. With PWeb, multiple-users can utilize the benefits of it from their own computers to prepare the initial data/parameters and access the simulation results without interrupting the Paramics simulation.

- As a part of Paramics: PWeb can be embedded within Paramics as an Agent/MIB of the SNMP external interface, which allows external software to communicate with a running simulation, to abstract data from it and to 
adjust parameters within it. This development would have to be undertaken by the Paramics' developer.

\section{The ImPLEMENTATION OF PWEB}

The development and implementation of PWeb requires the involvement of computer scientists, but transportation modelers will find the final Web interface more easy to use and also more efficient. The system requirements are basically the same as those currently required for Paramics, such as Window 2000 or later versions, but there are other requirements for a Web-based system, such as, Windows Explorer, Tomcat, the Java/JSP packet and a simple database system.

The PWeb approach may provide benefits for Paramics users, for example, as an alternative for the Application Programmer Interface (API) [2, 4, 9]. Actually, PWeb is also a special type of API, but with a web interface and internet communications. It also uses Java, which solves the problem of cross-platform application development. It is an advanced API development because of the advantages of the Web and Java applications.

\section{A. Preventing conflicts}

With the actual SNMP external interface, there can be some problems with the data being transmitted from controllers. Paramics can only receive the information from the controller,

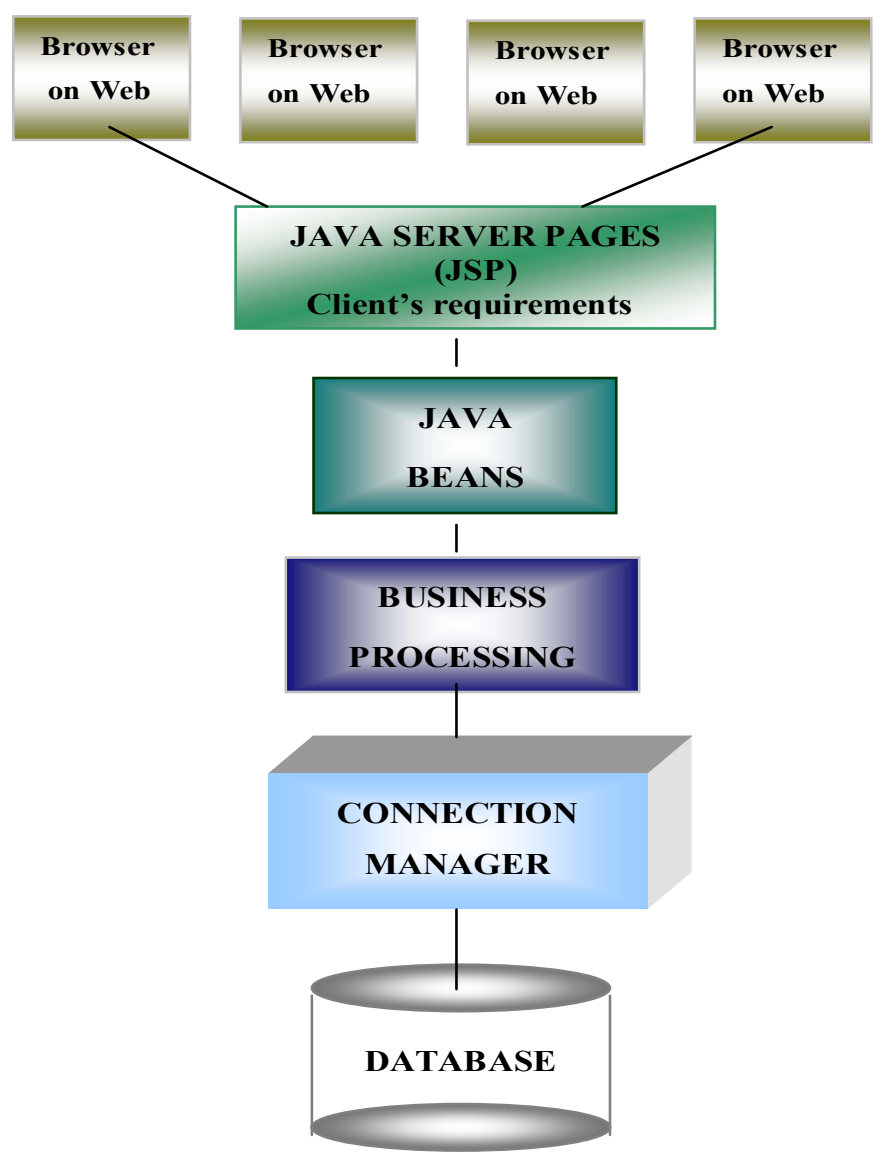

Fig. 3 Basic components of PWeb which is currently in direct communication. At the same time, the information from other controllers, which are also sending data at the same time will be lost. No indication will be provided to these sending controllers that the data was not received. This problem is solved by the development of PWeb.

To access PWeb from any controller, the problem can be resolved by using a dynamic Java/Web/Database system. A dynamic web site generates HTML/JSP pages to get the information and stores them in a database [5, 6]. Each request from a controller for the Web page requires an access of the database to be made. But connecting to a database is a time consuming activity since the database must allocate communication and memory resources as well as authenticate the user and set up the corresponding security context. Establishing the connection once and then use the same connection for subsequent requests can therefore dramatically improve the performance of a database driven web application. Since Java/Servlets can keep information between requests, a database connection pool provides a straight forward solution.

With this system, the information from controllers is received by PWeb and temporarily stored in a database within PWeb to wait for an available connection between the Paramics Agent/MIB and PWeb.

\section{B. The mechanism to manage priority}

After receiving and storing the information from the controllers, the problem now is to determine the sequence in which this information should be passed to the Paramicros Agent/MIB from the PWeb/database.

A rule-based mechanism has been established to manage the data processing in PWeb. Initially, the following rules were selected depending on the location of controllers and some unexpected events (the rules are stated in sequence of importance and application conflicts are resolved using this sequence).

- The importance of locations, such as the downtown area, the airport access roads, etc., where these locations may require greater priority than other areas;

- Events in the network, such as recent serious traffic congestion, accidents, flood or fire etc;

- Other locations with high traffic density;

- The first in first out rule.

\section{Components of the system}

The technological platform of PWeb is Web/NT/Tomcat. Sybase Central 4.0 is used as the database system. This system was also used in the control center for ITS applications [7]. Some basic concepts are explained in this section. The basic components of PWeb are shown in Fig. 3.

- Connection manager: is implemented as a data bank connection class allowing communication with the data bank. Initially, 20 channels were defined in the connection pool for communication. 
- Business processing: contains the application logic in which the classes to perform the calculation, transformation and organizer/query data bank etc. reside.

- JavaBeans: makes it possible to write component software in the Java programming language. Components are self-contained, reusable software units that can be visually composed into composite components, applets, applications, and servlets using visual application builder tools. The rule-based system is developed in this part.

- JavaServer Page (JSP): technology allows web developers and designers to rapidly develop and easily maintain, information-rich, dynamic web pages that leverage existing business systems. As part of the Java family, JSP technology enables rapid development of web-based applications that are platform independent.

\section{The IMPLEMENTATION OF PWEB: Model CALIBRATION CASE STUDY}

PWeb was developed to manage the information processing from the controllers to access Paramics SNMP Agent/MIB. As an example, $\mathrm{PWeb}$ is proposed to be applied to calibrate a Paramics model in the center of Calgary. Paramics is used as a kernel simulation tool in this research. When the simulation model was constructed, the main problem was to confirm the precision of the model. At this moment, the historical traffic data collected from 320 detect counts within the study area during 1999, 2000 and 2001 were used to calibrate the Paramics model. On the other hand, on-line calibration is also proposed as a complementary method. This paper concentrates on the on-line data processing. An initial version of PWeb was implemented to simulate the process of the traffic data collection from the loop detectors to calibrate the Paramics model.

\section{A. The controller}

The data from the intersection or link loop detectors within the simulated study area were used as an input to the Paramics model. The study area, in downtown Calgary, included $10 A v e, 11$ Ave, and 12Ave. The detector data is sent to the traffic control center 24 hours a day.

When considering the on-line calibration situation, four detector counts in every intersection are configured as a controller and the data is transmitted to Paramics every 15 minutes during a day, which is decided by the City of Calgary. To get the data from 80 controllers, it is necessary to develop an efficient system to manage communication and to resolve conflicts.

\section{B. $P W e b-a$ special controller}

PWeb is developed as a special controller. Firstly, it receives the traffic count data from the detectors. Secondly, it sends the data to the Paramics SNMP external interface. PWeb can receive the information from 20 controllers at the same time. If there are more than 20 controllers communicating with PWeb at the same time, the additional information can be stored in a buffer to await a free communication channel. When a connection is available, the information waiting to be processed is selected in a First In First Out manner to be stored in the PWeb database.

\section{The priority mechanism for communicating with Paramics}

From the PWeb database to the Agent/MIB, the information is processed using a common communication format between controller and Paramics. The detailed manner of communication is described in the Paramics SNMP Interface manual[3]. PWeb was developed to use priority access rules to get the data from the database to the channel to the Paramics Agent/MIB through SNMP. Paramics can use the traffic count data from detectors to estimate an O/D matrix [1]. In this research, the sequence of data processing from $\mathrm{PWeb}$ to Agent/MIB follows the creation of the matrix. For example, the priority to process the data starts with the first detector and then along the $10^{\text {th }}$ Ave, $11^{\text {th }}$ and then the last detector on $12^{\text {th }}$ Avenue. For every simulation segment of Paramics, the data is sent from $\mathrm{PWeb}$ for each designated time period, which in our example was every 15 minutes.

\section{The simulation}

Using the computer, for the developed PWeb simulation shown, the data processing procedure gets the data from the simulated detectors and transmits this data to $\mathrm{PWeb}$ and then it is stored in the database. PWeb also manages the sequence in which to send the data for use in Paramics. In a real simulation case, i.e. to get the data directly from the detectors to PWeb, it is necessary to configure the detectors as controllers in SNMP external interface standard [3]. In the future, this will depend on the need for cities to develop online microsimulation systems.

\section{The IMPLementation OF PWeB: Automatically ACCESSING SIMULATION RESULTS}

In this research PWeb has also been used to display the Paramics simulation results to multiple users. There are two ways to illustrate Paramics simulation results. The first one is to use the Paramics Visual Analyser tool to display statistics. In order to obtain more detailed quantitative results, another way is to abstract and display the results from text files in the Paramics host computer. The data in Table 1 is partly from a trace.txt file from a Paramics simulation on a section of a highway in Calgary:

TABLE I

TRACE .TXT FILE FROM A PARAMICS SIMULATION

\begin{tabular}{ccccc}
\hline \hline No & $\begin{array}{c}\text { Headway } \\
(\mathrm{s})\end{array}$ & $\begin{array}{c}\text { Distance } \\
(\mathrm{m})\end{array}$ & $\begin{array}{c}\text { Speed } \\
(\mathrm{km} / \mathrm{h})\end{array}$ & $\begin{array}{c}\text { Acceleration } \\
(\mathrm{m} / \mathrm{ss})\end{array}$ \\
\cline { 1 - 2 } 2 & 1.39 & 21.1 & 67.93 & -1.306 \\
1 & 1.56 & 272.2 & 61.01 & 0.000 \\
3 & 1.56 & 176.1 & 61.01 & 0.000 \\
2 & 1.39 & 16.4 & 66.31 & -1.805 \\
1 & 1.56 & 268.0 & 61.01 & 0.000 \\
3 & 1.56 & 171.8 & 61.01 & 0.000 \\
2 & 1.39 & 11.8 & 64.10 & -2.452 \\
1 & 1.56 & 263.7 & 61.01 & 0.000 \\
3 & 1.56 & 167.6 & 61.01 & 0.000 \\
$\ldots$ & $\ldots$ & $\ldots$ & $\ldots$ & $\ldots$ \\
\hline \hline
\end{tabular}


Deerfoot Trail from 16th Avenue to Beddington Trail [8]. In order to analyze the speed profile of vehicles, the user needs to go to the ../Log/run-00n subdirectory, read the .txt file, and transfer it to an Excel file.

Figure 4 shows the same data from the trace.txt file, automatically transferred by PWeb to a web page. This reduces the research procedure time and facilitates access by multiple users. They don't just have to use the Paramics host computer, but can use any Web connected computer to analyze the simulation results.

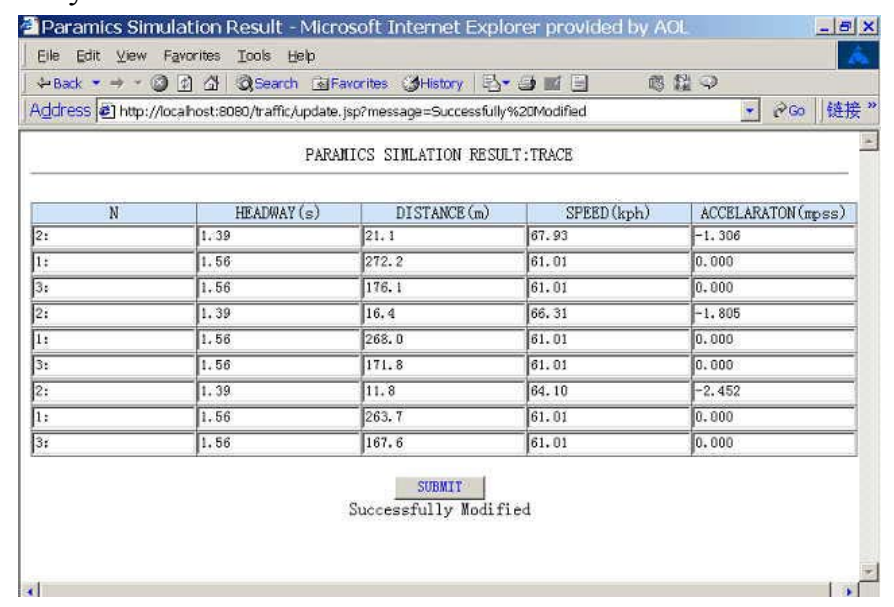

Fig. 4. Trace.txt file in Web.

\section{CONCLUSIONS}

We presented PWeb which was designed and developed to manage the communication between several traffic controllers and the Paramics SNMP external interface. We described the basic concepts and components of the system. This implementation allows transportation modelers to connect controllers with Paramics using the Web. It can manage the data processing from controllers to Paramics and resolves conflicts. After a simple adaptation, it can also process other information from UTC systems using alternative resources, such as video images, wireless, etc.

Initially, PWeb was implemented and only used to complete the communication function of Paramics SNMP external interface to prevent conflicts and to facilitate multiple-users applications. If the Paramics' developers accept this idea, it may be combined with the SNMP external interface within the Paramics software. In this case, the ability to communicate may be significantly improved. On the other hand, the quick development of Information Technology supplies the opportunities to improve the Internet based communication among the Paramics simulator and controllers. Web Services applications may be a perspective of next step research [12].

The PWeb application is at the computer simulation stage. The data was generated from simulated loop detectors and not the real counts, because the configuration of the detectors to the SNMP standard requires an enormous amount of work for a city transportation authority. In future, this will depend on the development of on-line microsimulation models.

\section{REFERENCES}

[1] SIAS, Paramics Reference Manual, Paramics 2002, reference manual, 2002.

[2] Quadstone Limited. http://www.paramics-online.com/index2.htm. Accessed January 2003.

[3] SIAS, Paramics External Interfaces, Paramics 2002 Alpha release, 2002.

[4] Lee, Der-Horng, P. Chandrasekar and Ruey-Long Cheu, Customized simulation modeling using Paramics Application Programmer Interface, Proceedings of 2001 IEEE Intelligente Transportation System Conference, pp. 842-847, Oakland (CA), USA, August, 25-29, 2001.

[5] Han Bergsten, Improved Performance with a Connection Pool,http://www.webdevelopersjournal.com/columns/connection_pool.h tml, Accessed January 2003.

[6] Jacobson, I., Booch, G. and Rumbaugh, J. (1999) The Unified Software Development Process, Addison Wesley Longman, Inc. USA.

[7] Weigang, L., Marlon Winston Koendjbiharie, Ricardo Cezar de Moura Jucá, Yaeko Yamashita, Andrew MacIver, "Algorithms for Estimating Bus Arrival Times Using GPS Data", Proc. of The IEEE 5th International Conference on Intelligent Transportation Systems, pp. 868873, September, 2002, Singapore.

[8] Basnayake, C., Gerard Lachapelle and Andrew MacIver, A GPS Based Calibration Tool for Microscopt Traffic Simulation Models, SMART MOVING Conference, April 2003.

[9] Ma, T. and Abdullhai, B., Genetic Algorithm-Based Combinatorial Parametric Optimization for the Calibration of Microscopic Traffic Simulation Models. Proceedings of 2001 IEEE Intelligente Transportation System Conference, pp. 848-853, Oakland (CA), USA, August, 25-29, 2001.

[10] Ziliaskopoulos, A.K. and S.T. Waller (2000), An Internet Based Geographic Information System that Integrates Data, Models and Users for Transportation Applications, Transportation Research, Part C, Vol. 8, pp.427-444.

[11] Weigang, L., Yaeko Yamashita, Marlon Winston Koendjbiharie, Ricardo Cezar de Moura Jucá, Deng XiJun, Osmar Quirino da Silva, Andrew MacIver, "The development and implementation of the system and data bank for the Intelligent Transportation System", 9th World ITS Congress, October 14-18, 2002, Chicago, USA.

[12] Wu, Chun-Hsin, Da-Chun Su, Justin Chang, Chia-Chen Wei, Kwei-Jay Lin, and Jan-Ming Ho, The Design and Implementation of Intelligent Transportation Web Services, in the Proceedings of IEEE Conference on Electronic Commerce, Newport Beach, California, USA, 2003. 